\title{
Development of a Silicon PIN Diode X-Ray Detector
}

\author{
Joshua Abramovitch ${ }^{\mathbf{1}}$ \\ Southern Methodist University \\ 3215 Daniel Avenue, Dallas, Texas, United States \\ E-mail: jkabramov@gmail.com \
}

\section{Chonghan Liu}

Southern Methodist University

3215 Daniel Avenue, Dallas, Texas, United States

E-mail: kentesmu.edu

\section{Tiankuan Liu}

Southern Methodist University

3215 Daniel Avenue, Dallas, Texas, United States

E-mail: I iulsmu. edu

\section{Jingbo Ye}

Southern Methodist University

3215 Daniel Avenue, Dallas, Texas, United States

E-mail: yejb@smu. edu

\section{Xiandong Zhao}

Southern Methodist University

3215 Daniel Avenue, Dallas, Texas, United States

E-mail: xiandongzesmu.edu

X-ray detectors currently on the market are expensive, costing thousands of dollars each and still limited in data acquisition options. The goal of this project is to create a device that can be used to accurately measure gamma and x-ray flux, calculate radiation dose rates, and be simple and inexpensive to produce. Developing an accurate and reliable system of measuring gamma and x-ray flux will allow for laboratories to conduct irradiation tests with confident calibration.

Many methods used to calculate x-ray flux are incapable of accounting for the high x-ray flux generated by modern X-ray machines. Advances in the technology of silicon PIN diodes in the datacom and telecom industries allows for the development of an inexpensive and compact solid-state ionizing sensor to calculate x-ray flux.

X-ray flux can be determined from the photocurrent produced by silicon PIN diodes when they capture a photon. The photocurrent is estimated for a given energy from the charge deposited, and x-ray flux in turn can be calculated from the current, charge, energy deposited, and properties of silicon.

1 Speaker 
Two working detector circuits, an AC-coupled version that outputs pulses and a DC-coupled version that outputs a DC voltage level, have already been completed. Cosmic muon, beta particle, and gamma photon events have been observed and characterized. Also, the charge and energy deposited onto the PIN photodiodes have been estimated. Remaining work for this project will be to combine the AC and DC circuits into one system with readout electronics.

Technology and Instrumentation in Particle Physics 2014

2-6 June, 2014

Amsterdam, the Netherlands 


\section{Introduction}

Many methods used to calculate $\mathrm{x}$-ray flux are incapable of accounting for the high $\mathrm{x}$-ray flux generated by modern x-ray machines. The most common method utilized today for monitoring x-ray dose rate in real time, by ionizing gas chambers, is usable for modern devices but is technically complex. The construction of a gas chamber is not a simple task, and its large size necessitates gas tubing, high voltage, signal cables, and other specialized parts and services to operate. The ionization approach is impractical for certain applications, for example when radiation dose must be measured in confined spaces. Advances in the technology of silicon PIN diodes in the datacom and telecom industries allows for the development of an inexpensive and compact solid-state ionizing sensor to calculate $\mathrm{x}$-ray flux [1]. As x-ray photons flux through the diodes, the photoelectric effect produces a photocurrent. The x-ray flux can be determined from this current, and once the former is known the radiation dose can be calculated from it.

\section{Characterization of $\beta, \gamma$, and $\mu$ Events}

To establish a theoretical foundation, particle impact events on the silicon PIN photodiode were observed and the charge and energy deposited were estimated. Most of the experiments conducted at this stage utilized commercially-available devices, a Cremat CR-110 chargesensitive preamplifier (CSP) and a Cremat CR-200 Gaussian shaping amplifier (GSA). The gain of the GSA was set to 10, and the gain of the CSP was $1.4 \mathrm{~V} / \mathrm{pC}$. The amplification of the whole system was $134.4 \mathrm{~V} / \mathrm{pC}$. Although expensive and requiring two stages, the commercial devices produced clean output and allowed for the observation of said events via digital oscilloscope. According to the test results, the system noise was very low, on the order of 10 $\mathrm{mV}$. The noise threshold chosen for triggering was $130 \mathrm{mV}$, approximately 10 standard deviations above the mean system noise value of $30 \mathrm{mV}$.

Initially, the objective was to compare the signals produced by alpha $(\alpha)$, beta $(\beta)$, and gamma $(\gamma)$ button sources. Figures 1 and 2 below show the pulse height distributions for $\beta$ and $\gamma$ events. No $\alpha$ events were observed because $\alpha$ particles could not penetrate the plastic housing of the diodes.

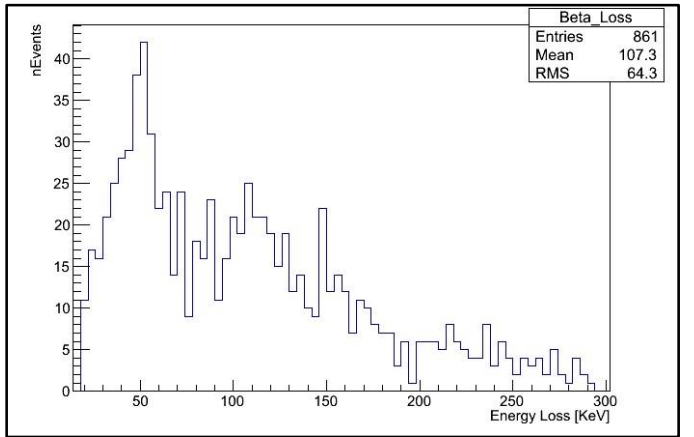

Figure $1-\beta$ Event Energy Distribution

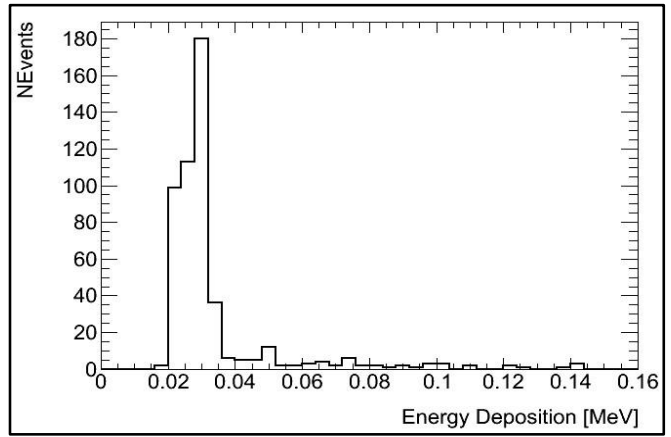

Figure $2-\gamma$ Event Energy Distribution 
Once the data was collected, the Bethe formula was used to calculate the $\mathrm{dE} / \mathrm{dx}$ for $\beta$ events [2]. The result of this expression is a $\mathrm{dE} / \mathrm{dx}$ value of $3.599 \mathrm{MeV} / \mathrm{cm}$. This value was inserted into Equation 1:

$$
\Delta x=\frac{\Delta E_{\beta}}{d E / d x}=0.3 \mathrm{~mm}
$$

where $\Delta \mathrm{E}_{\beta}$ is $0.1073 \mathrm{MeV}$ for silicon with electron kinematic energy $\left(\mathrm{E}_{\mathrm{k}}\right) 2.0 \mathrm{eV}$. This value is identical to that of ESTAR, a database cataloguing the stopping power of electrons in various materials [4].

The charge deposited for an average $\beta$ event was calculated by dividing the mean $\beta$ event output voltage $(638 \mathrm{mV})$ by the system amplification resulting in a $\mathrm{Q}_{\beta}$ of $4.747 \mathrm{fC}$. This value was then inserted into Equation 2:

$N_{e}=\frac{Q_{\beta}}{Q_{e}}=2.96319 \times 10^{-4}$

where $\mathrm{Q}_{\mathrm{e}}$ is the electron charge $\left(1.602 * 10^{-19} \mathrm{C}\right) . \mathrm{N}_{\mathrm{e}}$ was then utilized in Equation 3:

$\Delta E_{\beta}=N_{e}\left(I E_{S i}\right)=0.1073 \mathrm{MeV}$

where $\mathrm{IE}_{\mathrm{Si}}$ is the silicon ionization energy $(3.62 \mathrm{eV})$. This value corresponds to the mean $\beta$ energy loss in ${ }^{90} \mathrm{Sr}$ [5].

The thickness of the silicon in the PIN diodes was then estimated to verify the accuracy of the $\Delta \mathrm{E}$ calculations. $\mathrm{E}_{\mathrm{k}}$ for silicon ranges from $0.2 \mathrm{MeV}$ to $2.0 \mathrm{MeV}$; from ESTAR, this corresponds to a $\mathrm{dE} / \mathrm{dx}$ range of $3.651 \mathrm{MeV} / \mathrm{cm}$ to $5.231 \mathrm{MeV} / \mathrm{cm}$. Therefore, $\Delta \mathrm{x}$ ranges from $0.205 \mathrm{~mm}$ to $0.306 \mathrm{~mm}$. According to the data sheet for the PIN diodes used, the silicon is $0.28 \mathrm{~mm}$ thick [6]. Therefore, the above calculations can be considered accurate.

The system was also left running for 3 hours to characterize cosmic $\mu$ interactions. The energy deposition distribution is shown in Figure 4. The majority of events were about 0.05 $\mathrm{MeV}$ in energy.

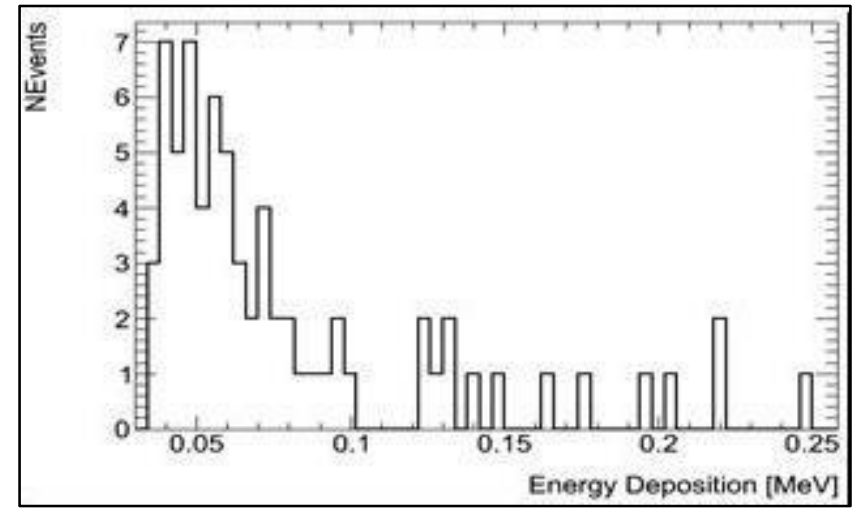

Figure $4-\mu$ Event Energy Distribution 


\section{Comparison to GEANT4 Simulations}

Simulations were run in GEANT4 for comparison with the calculations and experimental data. A simulation for $\gamma$ events due to interactions with a ${ }^{133}$ Ba source are shown in Figure 5(a).

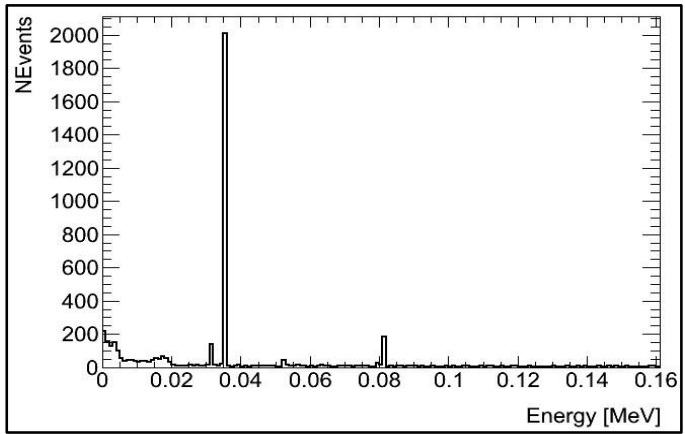

Figure 5(a) - Simulated $\gamma$ Event Distribution

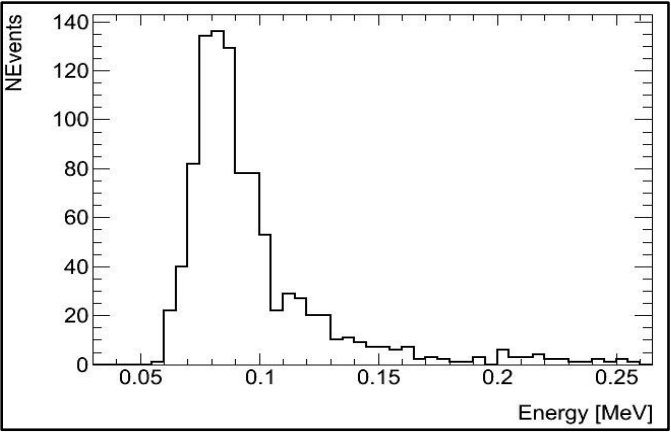

Figure 5(b) - Simulated $\mu$ Event Distribution

The energy deposition peaked sharply at a mean of $0.04452 \mathrm{MeV}$. This is close to the experimental results shown in Figure 2, which exhibited a mean of approximately $0.03 \mathrm{MeV}$ with a distribution width of approximately $0.0075 \mathrm{MeV}$. As such, the GEANT4 simulation conforms to the actual physical process in the PIN diode.

A simulation for cosmic $\mu$ events resulted in a mean energy deposition of approximately $0.09 \mathrm{MeV}$, as shown in Figure 5(b). In the experimental data, however, the mean energy deposition was closer to $0.05 \mathrm{MeV}$. For the experimental data, however, fewer than one hundred samples for $\mu$ events have been collected thus far. As such, more samples are needed to compare the experimental and simulation results.

\section{Circuit Design and Prototypes}

The first type of circuit developed was AC-coupled, intended to produce pulses for use as a pulse counter. The basic design is based on a design submitted to Radiation-Watch.org, a non-profit project initiated after the Fukushima Daiichi nuclear disaster, for a compact radiation counter that can be used with an iPhone [7].

Up to eight PIN photodiodes are placed in reverse bias, with the positive end connected to the first amplification stage. When a gamma ray strikes one of the photodiodes, a photocurrent is produced. The current then goes through two stages of transimpedance amplification to produce an output voltage. The capacitors immediately after each op-amp serve to eliminate DC offset. As a result, the circuit only registers pulses when a photon interacts with a photodiode. Additional resistors and capacitors are used to filter the output and reduce noise.

An AC prototype was placed in a black, light-tight plastic, connected to a $9 \mathrm{~V}$ battery, and tested in Brookhaven National Laboratory's gamma irradiation chamber. In this dark, quiet environment, the output registered pulses for incident gamma rays from a button source at a low dose rate. As shown in Figure 6, the pulses were on the order of $10 \mathrm{mV}$. Placing the circuit close to the chamber's ${ }^{60} \mathrm{Co}$ gamma source for a higher dose rate resulted in many overlapping pulses of average amplitude $40 \mathrm{mV}$. The average energy of the ${ }^{60} \mathrm{Co}$ gamma rays was $1.25 \mathrm{MeV}$, and the average energy of the button source's gamma rays was $356 \mathrm{keV}$. 


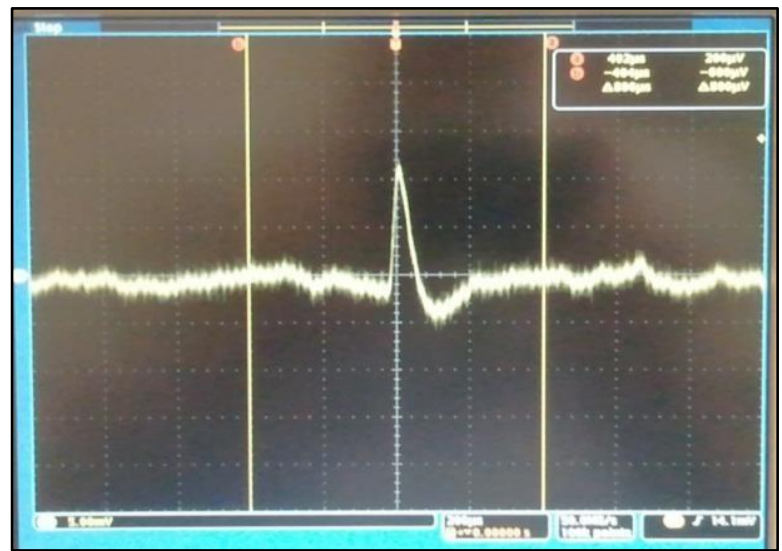

Figure 6 - AC Prototype 4 Output for a Low Dose Rate (Button Source)

\section{Future Work}

The next step in the project is to develop a low-cost multi-channel readout system and build a teaching apparatus of a sampling electromagnetic calorimeter. To this end, a ST Microelectronics STM32F051R8T6 microcontroller is being programmed. Also, the circuits are to be redesigned with transimpedance amplifiers sourced from the telecom industry. This will allow for a higher-quality circuit without a large increase in overall cost. Also, GEANT4 will be used for additional simulations of $\beta, \gamma$, and $\mu$ events, as well as to simulate the redesigned circuits and calorimeter system. In addition, the final system will be calibrated by measuring the dose rate of an X-RAD iR-160 cabinet $\mathrm{X}$-ray irradiator with a standard dosimeter.

\section{References}

[1] Owen, Robin L., et al. Determination of X-Ray Flux Using Silicon Pin Diodes. Journal of Synchrotron Radiation 16.2 (2009): 143-51.

[2] X. Wang, Particle Detection Technology [M], Hefei: USTC, 2009.

[3] Richard C. Fernow, Introduction to experimental particle physics, Cambridge: Cambridge University, 1986.

[4] International Commission on Radiation Units and Measurements. ICRU Report 37, Stopping Powers for Electrons and Positrons.

[5] E. N. Jensen and L. Jackson Laslett, Physical Review, vol. 75, Issue 12, pp. 1949-1950

[6] Vishay Semiconductors, VBPW34FAS, VBPW34FASR Silicon PIN Photodiode, 11 August 2011, http://www.vishay.com/docs/81127/vb pw34fa.pdf.

Radiation-Watch.org. 2011. 14 Apr. 2014, http://www.radiation-watch.org/p/english.html 
